$\stackrel{N / 2}{N}$

Global Journals Inc

(8)

\title{
Exploring Effective Factors in the Demographic and Motivation Issues of the Banking Sector of Bangladesh
}

\author{
By Mohammad Azizur Rahman
}

Begum Rokeya University

Abstract- The paper attempted to explore the most effective factors of demographic outlined affecting employees' motivation. With a view to empirical analysis data were collected from 400 bank employees. In the demographic issues gender, age, experience, pay, type and name of the bank, present and prior designations etc. were considered. For inferential statistics there were five regression models with ANOVA and coefficients models developed in the study. The dependent variables were namely work experience, present basic and gross salary, and initial basic and gross salary and 94\%, 77\%, 68\%, 48\% and 35\% respectively explained by other independent predictors. The study found that work experience was strongly affected by age. On the other hand, present pay (basic and gross) was highly subjective by present designation and initial basic and gross salaries were prejudiced by initial or first joining designation of the employees. The study was also observed on findings that age, present and first joining designations modify employees' motivation in the banking sector of Bangladesh (showed in Table 18).

Keywords: age, gender, work experience, initial salary, gross salary, motivation.

GJMBR-A Classification: JEL Code: M12

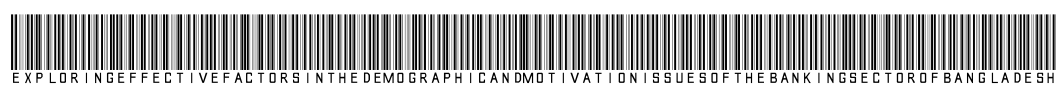

Strictly as per the compliance and regulations of:

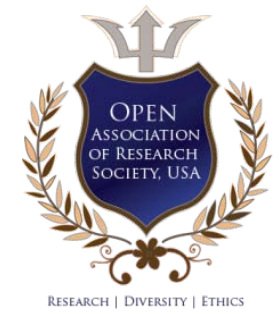

(C) 2020. Mohammad Azizur Rahman. This is a research/review paper, distributed under the terms of the Creative Commons Attribution-Noncommercial 3.0 Unported License http://creativecommons.org/licenses/by-nc/3.0/), permitting all non-commercial use, distribution, and reproduction in any medium, provided the original work is properly cited. 


\title{
Exploring Effective Factors in the Demographic and Motivation Issues of the Banking Sector of Bangladesh
}

\author{
Mohammad Azizur Rahman
}

\begin{abstract}
The paper attempted to explore the most effective factors of demographic outlined affecting employees' motivation. With a view to empirical analysis data were collected from 400 bank employees. In the demographic issues gender, age, experience, pay, type and name of the bank, present and prior designations etc. were considered. For inferential statistics there were five regression models with ANOVA and coefficients models developed in the study. The dependent variables were namely work experience, present basic and gross salary, and initial basic and gross salary and $94 \%, 77 \%, 68 \%, 48 \%$ and $35 \%$ respectively explained by other independent predictors. The study found that work experience was strongly affected by age. On the other hand, present pay (basic and gross) was highly subjective by present designation and initial basic and gross salaries were prejudiced by initial or first joining designation of the employees. The study was also observed on findings that age, present and first joining designations modify employees' motivation in the banking sector of Bangladesh (showed in Table 18).
\end{abstract}

Keywords: age, gender, work experience, initial salary, gross salary, motivation.

\section{Introduction And Review Context}

uman Resource Management (HRM) is regarded as the significant department for talent management. The talented employees compulsorily considered as assets in the organization which can be effective through acquisition, development, motivation and maintenance integrated from all units of the bank. HRD selects the right kind of personnel for the committed posts. In addition to this, HRD maintains talent human resources for the bank by applying various motivational techniques and HRs considered as soft assets (www.ventureline.com). Since bank is a service industry, this is why, its sustainability and competitive opportunities depends on how HR Department utilize their potentialities and competencies. The need of talent employees is well recognized in all events of global financial crisis (2007-2008) that was mostly connected with ambitious and ineffectual investment decision by the banks (Islam et al, 2017). Bangladesh Bank (BB) and Ministry of Finance (MoF) emphasized to ensure better HRM in the banking sector of Bangladesh through circulars (BB \& MoF, 2015). The

Author: PhD Student, IBS, RU \& Associate Professor, Management Studies Begum Rokeya University, Rangpur, Bangladesh.

e-mail: azizru07@gmail.com circulars are associated with rules and regulations, maintaining banking hours, salaries and incentives, leave and performance appraisal of female employees.

Motivation means inspiring people to work continuation. Motivated employees do have best quality of performance. Employees' motivation is affected by various factors. The total environment of the banking industry has become changed due to face the competitive sustainability. Basic pay, gross pay, designation, promotion, scoring of banking diploma for promotion, experiences, location or posting, types of bank etc. are the issues of demographic factors. Designation and gross pay are highly correlated for motivation.

Different emerging theories of motivation support that age, experience, pay, designation etc. affect the motivation. Work experience relates the holding position through up gradation in the organization. Maslow's need hierarchy theory proves the said issues of demographic (Maslow, 1954). On the other supportive part of the Two-Factor Model namely Hygiene and Motivating factors. Employees can enjoy these factors related to opportunities over a long-term service the organization (Herzberg, 1959, 1987). The Two Factor Theory states that human needs are ordered in a series of levels of the hierarchy accordance to importance which creates motivation. Alderfer's (1969) ERG theory chains Need-Hierarchy and Two-Factor Theory of motivation. Existence (E) chains the basic need and job security, relatedness (R) actually related to social needs and growth $(G)$ is supportive with status and highest level achievement. If a higher order need constants or unfulfilled, an individual can degenerate to lower order needs which cause satisfy easily. Mc Clellend's Three Needs Model (1961) is also supportive to Needs Theory of Maslow whereas it can be seen that need for affiliation (social need), need for power related to ego/status and remaining one that is need for achievement connected to challenging achievement. An employer does have the ability to understand the needs so as to meet these needs having a better chance of involving and retaining talent employees. A survey conducted by Watson Wyatt Worldwide showed that $71 \%$ people deliberate money as the main reason to stay as followed by promotion prospect (33\%) (Endress, 2007). Abraham Harold Maslow suggested a theory that 
delineated five hierarchical needs which could also be functional to an organization and its employees' performance (Gordon, 1965). Without one of the lower ones the second need could be achieved or satisfied. Maslow's needs theory is static imperative and applicable in today's business organizations, for every organization that seek to obtain success and excellence reflects positively the organizational culture, HRM and the employee's productivity, to attain organizational excellence and create good environment, better and enjoyable work environment and achieve goal at the right time then a effort and application of the theory is supreme (Jerome, 2013). When an organization tries to know what drives personnel to work more, it is in a better position to influence them to perform well (Kovach, 1987). Again it clarifies that employees performance can be examined by three indicators namely ability, workplace environment, and motivation (Griffin, 1990). It is critical job for the managers in terms of motivating subordinates since it is noticed that there globally changes in demographic factors, as well as highly advanced technology (Wiley, 1997). This only emphasized the need to explore what motivates employees in order to get better performance. The author highlighted the most and less important factors of motivation in workplace from the earlier management. The most important factors in 1946 (appreciation), 1980 (interesting work), 1986 (interesting work) and 1992 (good wages) and less important factors were in 1946 (discipline), 1980 (discipline), 1986 (personal problems), and 1992 (personal problems). The study received the most striking findings that money and job security are the clear indicators of motivation. The effective pay program, a primary motivating factor is critical due to individuals and psychological possessions. It is does have immaterial value of the reward, but the increase in self-regard that public recognition accompanying with monetary compensation affords (Dawson and Dawson, 1990). In the past, huge numbers of research studies on employee motivation in the different perspectives already conducted. Very few of them were conducted relating to demographic composition with motivation. The present paper stressed to observe the influential factors of motivation with the sources variables of demographic issues among the employees in the sampled banks. The study also designed to explore the most effective factors in motivation.

Research questions: Does demographic composition affect employee motivation in the banking sector?

Objective of the study: The main objective of the study was to scan effective factors in demographic and motivating focuses among the employees of the banking sector in Bangladesh.

\section{Research Framework}

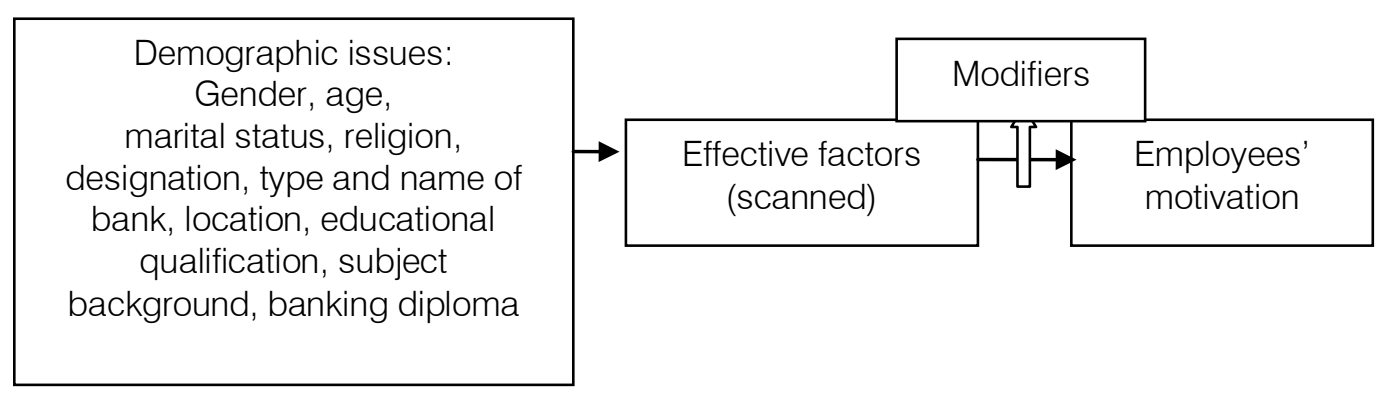

\section{Hypotheses}

Alternative hypotheses $\left(\mathrm{H}_{\mathrm{a}}\right.$

$H_{a 1} \quad$ Employee motivation is related to work experience in the sampled banks.

$H_{a 2}$ There is a positive relationship between employee motivation and present basic salary

$H_{\text {aз }}$ Present gross salary affects employee motivation in the banking sector.

$H_{a 4}$ Initial basic salary motivates employees positively.

$H_{a 5}$ Initial gross salary motivates employee positively.

\section{METHODOLOGY}

The study has been designed based on empirical and quantitative materials. The employees who were in the service full time in the banks considered as participants. The primary data were collected during
March-June 2019 from 400 employees (320 employees of four public and remaining 80 employees of six private banks operated in the Northern three districts of Rangpur Division Bangladesh through sample random sampling (SRS). Data have been analyzed by SPSS IBM Version 22.0. Secondary data were collected from different research articles, published and unpublished books, dailies and websites. 


\section{Results and Discussion}

Table 1: Demographic outline of the respondents (source: Field survey, up to June 2019)

\begin{tabular}{|c|c|c|c|c|c|}
\hline \multicolumn{2}{|c|}{ Demographic issues } & Percent & Mean & SD & $\mathrm{CV}$ \\
\hline \multirow{2}{*}{ Sex } & Male & 83.5 & \multirow{2}{*}{1.165} & \multirow{2}{*}{0.372} & \multirow{2}{*}{$31.93 \%$} \\
\hline & Female & 16.5 & & & \\
\hline \multirow{3}{*}{ Age group } & $<30-40$ years & 59.5 & \multirow{3}{*}{2.633} & \multirow{3}{*}{0.922} & \multirow{3}{*}{$35 \%$} \\
\hline & $41-50$ years & 13.8 & & & \\
\hline & $>50$ years & 26.8 & & & \\
\hline \multirow{2}{*}{ Marital status } & Married & 99.0 & \multirow{2}{*}{1.010} & \multirow{2}{*}{0.100} & \multirow{2}{*}{$9.86 \%$} \\
\hline & Single & 1.0 & & & \\
\hline \multirow{2}{*}{ Religion } & Islam & 89.3 & \multirow{2}{*}{1.110} & \multirow{2}{*}{0.321} & \multirow{2}{*}{$18.94 \%$} \\
\hline & Hindu & 10.75 & & & \\
\hline \multirow{2}{*}{ Type of bank } & Public & 80.0 & \multirow{2}{*}{1.200} & \multirow{2}{*}{0.401} & \multirow{2}{*}{$33.38 \%$} \\
\hline & Private & 20.0 & & & \\
\hline \multirow{3}{*}{ Location (District) } & Rangpur & 41.5 & \multirow{3}{*}{1.743} & \multirow{3}{*}{0.712} & \multirow{3}{*}{$40.88 \%$} \\
\hline & Dinajpur & 42.8 & & & \\
\hline & Nilphamari & 15.8 & & & \\
\hline \multirow{2}{*}{$\begin{array}{l}\text { Educational } \\
\text { Qualification }\end{array}$} & Bachelor & 17 & 2660 & 0749 & $2815 \%$ \\
\hline & Master & 83 & 2.000 & 0.170 & 20.1070 \\
\hline & Science & 31.8 & & & \\
\hline Discipline/ Group & Humanities & 50 & 1.865 & 0.695 & $37.26 \%$ \\
\hline & Business & 18.3 & & & \\
\hline & $\mathrm{Nil}$ & 62.3 & & & \\
\hline Bank Diploma & Part-1 & 21.3 & 0.543 & 0.761 & $140.34 \%$ \\
\hline & Part-2 & 16.5 & & & \\
\hline & $1-5$ & 11.3 & & & \\
\hline & $6-10$ Years & 45.5 & & & \\
\hline (Year) & 11-15 Years & 8.0 & 2.985 & 1.483 & $49.70 \%$ \\
\hline & 16-20 Years & 4.0 & & & \\
\hline & 20 Years (more) & 31.3 & & & \\
\hline Present Designation/ & Entry Level Officer & 77.4 & 5505 & 1319 & $23.96 \%$ \\
\hline Post & Executive Level Officer & 22.9 & & & \\
\hline Present Basic Pay & $<10000-300000 /-$ & 49.0 & 35512 & 14246 & $40.12 \%$ \\
\hline (Tk.) & 30000/- (more) & 511.0 & ك & & \\
\hline & $<20000-40000 /-$ & 19.0 & & & \\
\hline (TK) & $>40000-60000 /-$ & 38.0 & 60945 & 26809 & $44 \%$ \\
\hline & 60000/- (more) & 43.0 & & & \\
\hline First Joining & Entry Level Officer & 99.5 & 4.110 & 1925 & $46.85 \%$ \\
\hline Designation & Executive Level Officer & 0.5 & 4.110 & & \\
\hline Initial Basic Pav (Tk) & $<10000-20000 /-$ & 96.6 & 35512 & 14246 & $40.12 \%$ \\
\hline IItial Daste T ay (Th.) & $>20000-30000 /-$ & 3.5 & 30012 & $14<40$ & $40.12 \%$ \\
\hline Initial Groç Pay (Tk ) & $<20000-40000 /-$ & 98.8 & 13401 & 16433 & $12263 \%$ \\
\hline Initial Gross ray (IK.) & 40000-60000/- (more) & 1.2 & 13401 & 10433 & $122.63 \%$ \\
\hline
\end{tabular}

Regression Models (Table 2, 5, 8, 11, \& 14)

Table 2: Regression: Model Summary

\begin{tabular}{|l|c|c|c|c|c|c|c|c|c|}
\hline \multirow{2}{*}{ Model } & $\mathrm{R}$ & R Square & \multirow{2}{*}{$\begin{array}{c}\text { Adjusted R } \\
\text { Square }\end{array}$} & $\begin{array}{c}\text { Std. Err. } \\
\text { of } \\
\text { Estimates }\end{array}$ & $\mathrm{R}^{2}$ Change & $\mathrm{F}$ & $\mathrm{df1}$ & $\mathrm{df2}$ & Sig. F \\
\hline 1 & $0.971^{\mathrm{a}}$ & 0.942 & 0.940 & 2.741 & 0.94 & 483.94 & 13 & 386 & 0.000 \\
\hline
\end{tabular}


Remarks: The fitted linear regression model for working experience (year) and other independent variables listed. The model is good fit for this dataset and the coefficient of multiple determinations $R^{2}$ is 0.942 (Table
2). Since the $R^{2} \sim 0.94$ then the dependent variable work experience $94 \%$ explained by the independent variables/predictors.

Table 3: ANOVA ${ }^{a}$

\begin{tabular}{|c|c|c|c|c|c|c|}
\hline \multicolumn{2}{|r|}{ Model } & Sum of Squares & df & Mean Square & $\mathrm{F}$ & Sig. \\
\hline \multirow{3}{*}{1} & Regression & 47261.760 & 13 & 3635.520 & 483.944 & $0.000^{b}$ \\
\hline & Residual & 2899.737 & 386 & 7.512 & & \\
\hline & Total & 50161.498 & 399 & & & \\
\hline \multicolumn{7}{|c|}{ i. } \\
\hline \multicolumn{7}{|c|}{$\begin{array}{l}\text { b. Predictors: (Constant), Banking Diploma, Group/ Subject, Gender, Religion, Educational Qualification, Marital Status, } \\
\text { Location (District), Type of Bank, Present Designation, First joining designation/post, Name of the Bank, Age, Location } \\
\text { (Upazilla). }\end{array}$} \\
\hline
\end{tabular}

Hypothesis: The null hypothesis, $\mathrm{H}_{0}: \mathrm{B}_{0}=\mathrm{B}_{1}=\mathrm{B}_{2}=\ldots \ldots \ldots=\mathrm{B}_{13}=0$

The alternative hypothesis, $H_{1}: B_{0}=B_{1}=B_{2}=\ldots \ldots \ldots=B_{13} \neq 0$

Remarks: From the Table 3 ANOVA table the fitted regression model F-test statistic value is 483.944 and the significance value ( $p$ value) 0.000 which is less than $0.05(p$ value $<\alpha)$. Then all the regression coefficients were statistically highly significant at 5\% level of significance. That is the null hypothesis is rejected and alternative hypothesis is accepted. Therefore, all the regression coefficients were not zero (0).

Table 4: Coefficients ${ }^{a}$

\begin{tabular}{|c|c|c|c|c|c|c|}
\hline & \multirow{2}{*}{ Model } & \multicolumn{2}{|c|}{ Unstandardized Coefficients } & \multirow{2}{*}{$\begin{array}{c}\begin{array}{c}\text { Standardized } \\
\text { Coefficients }\end{array} \\
\text { Beta } \\
\end{array}$} & \multirow{2}{*}{$\mathrm{t}$} & \multirow{2}{*}{ Sig. } \\
\hline & & $\mathrm{B}$ & Std. Error & & & \\
\hline \multirow{14}{*}{1} & (Constant: Experience) & $-16.110\left(\mathrm{~B}_{0}\right)$ & 2.519 & & -6.395 & 0.000 \\
\hline & Gender & $0.664\left(\mathrm{~B}_{1}\right)$ & 0.402 & 0.022 & 1.652 & 0.099 \\
\hline & Age & $0.800\left(\mathrm{~B}_{2}\right)$ & 0.034 & 0.663 & 23.649 & 0.000 \\
\hline & Marital status & $0.697\left(\mathrm{~B}_{3}\right)$ & 1.417 & 0.006 & 0.492 & 0.623 \\
\hline & Religion & $-0.535\left(\mathrm{~B}_{4}\right)$ & 0.439 & -0.015 & -1.220 & 0.223 \\
\hline & Present designation & $1.294\left(B_{5}\right)$ & 0.181 & 0.152 & 7.150 & 0.000 \\
\hline & First joining post & $-1.663\left(\mathrm{~B}_{6}\right)$ & 0.147 & -0.286 & -11.334 & 0.000 \\
\hline & Type of Bank & $-0.567\left(\mathrm{~B}_{7}\right)$ & 0.636 & -0.020 & -0.891 & 0.374 \\
\hline & Name of the Bank & $-0.192\left(B_{8}\right)$ & 0.113 & -0.039 & -1.697 & 0.091 \\
\hline & Location (District) & $0.341\left(B_{q}\right)$ & 0.665 & 0.020 & 0.512 & 0.609 \\
\hline & Location (Upazilla) & $-0.055\left(B_{10}\right)$ & 0.098 & -0.023 & -0.555 & 0.579 \\
\hline & Educational qualif. & $-0.860\left(B_{11}\right)$ & 0.257 & -0.057 & -3.343 & 0.001 \\
\hline & Group/Subject & $0.001\left(\mathrm{~B}_{12}\right)$ & 0.206 & 0.000 & 0.005 & 0.996 \\
\hline & Banking Diploma & $0.013\left(\mathrm{~B}_{13}\right)$ & 0.223 & 0.001 & 0.059 & 0.953 \\
\hline
\end{tabular}

The fitted regression model can be defined as,

\section{Workingexperience(year)}

$$
\begin{aligned}
& =-16.110 *(\text { Constant })+0.664 * \text { Gender }+0.800 * \text { Age }+0.697 * \text { MaritalStatus }-0.535 \\
& * \text { Religion }+1.294 * \text { Presentdesignation }-1.663 * \text { Firstjoiningdesignation }-0.567 \\
& * \text { TypeofBank }-0.192 * \text { NameoftheBank }+0.341 * \text { Location }(\text { Disrtict })-0.055 \\
& * \text { Location }(\text { Upazilla })-0.860 * \text { Educationalqualification }+0.001 * \text { GrouporSubject }+0.013 \\
& * \text { BankingDiploma }
\end{aligned}
$$

Remarks: From the fitted (Table 4) the age, present designation, first joining designation, educational qualification were statistically significance at $5 \%$ level of significance. Therefore, employees' motivation is exaggerated by the control variable namely age, designation, and educational qualification. The subject or major discipline does not directly affect motivation $\left(\beta_{12}\right)=0.001$ and $\mathrm{p}$ value is 0.996 . Among the significant independent predictors age (0.800) and present designation (1.294) were positive contribution on the dependent variable working experience (year). 

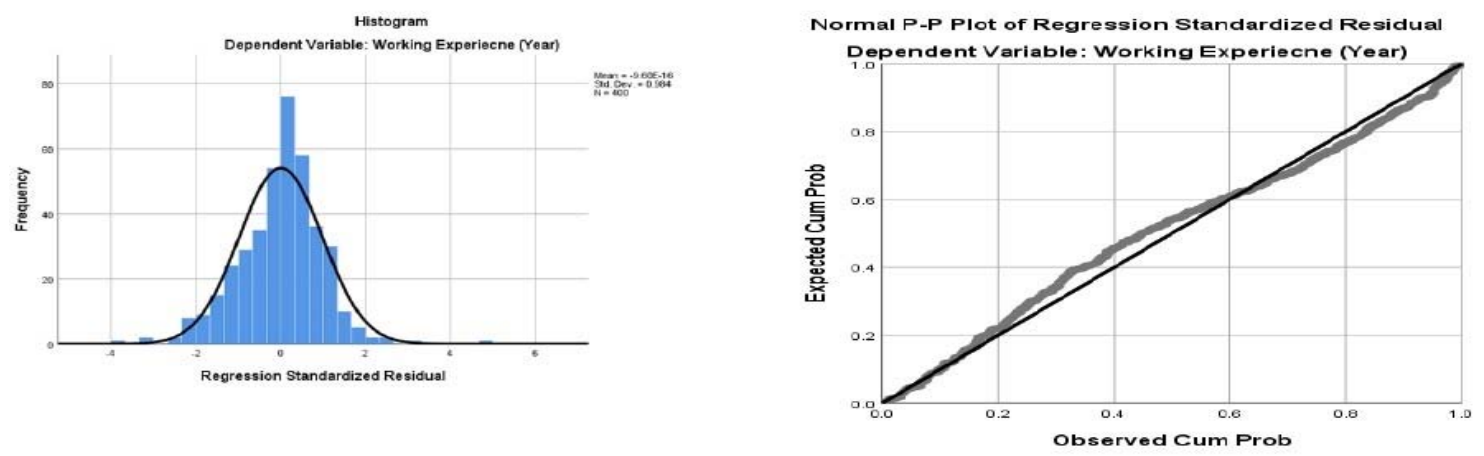

Figure 1 \& 2: Histogram and P-P Plot of Regression Standardized Residual (Experience)

Remarks: The Figure 1 \& 2 depict that the fitted histogram showed the bell shape curve and $Q-Q$ (quantile-quantile) plot the fitted residual line passing through the origin. Therefore, the dependent variable working experience (year) is normally distributed and the linear regression model best fit for this dataset.

Table 5: Regression-Model Summary

\begin{tabular}{|c|c|c|c|c|c|c|c|c|c|}
\hline \multirow[b]{2}{*}{ Model } & \multirow[b]{2}{*}{$\mathrm{R}$} & \multirow[b]{2}{*}{$\mathrm{R}^{2}$} & \multirow[b]{2}{*}{ Adjusted $\mathrm{R}^{2}$} & \multirow{2}{*}{$\begin{array}{l}\text { Std. Err. of } \\
\text { the } \\
\text { Estimates }\end{array}$} & \multicolumn{5}{|c|}{ Change Statistics } \\
\hline & & & & & $\mathrm{R}^{2}$ & $\begin{array}{c}F \\
\text { change }\end{array}$ & df1 & df2 & Sig. $F$ \\
\hline 1 & $0.877^{a}$ & 0.769 & 0.762 & 6987.86 & 0.769 & 99.1 & 13 & 386 & 0.000 \\
\hline
\end{tabular}

a. Predictors: (Constant), Banking Diploma, Group/ Subject, Gender, Religion, Educational Qualification, Marital Status, Location (District), Type of Bank, Present Designation, First joining designation/post, Name of the Bank, Age, Location (Upazilla).

a. Dependent Variable: Present Basic Salary.

Remarks: The above fitted linear regression model (Table 5) for present basic salary and other independent variables listed. The model is good fit for this dataset and the coefficient of multiple determinations $R^{2}$ is
0.769. Since the $R^{2} \sim 0.769$ then the dependent variable present basic salary $76.9 \%$ or about $77 \%$ explained by the independent variables/predictors mentioned above.

Table 6: ANOVA ${ }^{a}$

\begin{tabular}{|c|c|c|c|c|c|c|}
\hline \multicolumn{2}{|c|}{ Model } & Sum of Squares & df & Mean Square & F & Sig. \\
\hline \multirow{3}{*}{1} & Regression & 62872948092.258 & 13 & 4836380622.481 & 99.045 & $0.000^{b}$ \\
\cline { 2 - 7 } & Residual & 18848472031.68 & 386 & 48830238.424 & & \\
\cline { 2 - 7 } & Total & 81721420123.94 & 399 & & \\
\hline \multicolumn{7}{|c|}{ a. Dependent Variable: Present Basic Salary } \\
\hline \multicolumn{2}{|l|}{$\begin{array}{l}\text { b. Predictors: (Constant), Banking Diploma, Group/ Subject, Gender, Religion, Educational Qualification, Marital Status, } \\
\text { Location (District), Type of Bank, Present Designation, First joining designation/post, Name of the Bank, Age, Location } \\
\text { (Upazilla). }\end{array}$}
\end{tabular}

Hypothesis: The null hypothesis, $\mathrm{H}_{0}: \mathrm{B}_{0}=\mathrm{B}_{1}=\mathrm{B}_{2}=\ldots \ldots \ldots=\mathrm{B}_{13}=0$

The alternative hypothesis, $H_{1}: B_{0}=B_{1}=B_{2}=\ldots \ldots \ldots=B_{13} \neq 0$

Remarks: From the (Table 6) ANOVA (Analysis of statistically strongly significant at 5\% level of Variance) table the fitted regression model F-test statistic value is 99.045 and the significance value $(p$ value) 0.000 . Then all the regression coefficients were significance. That is alternative hypothesis is accepted. Therefore, all the regression coefficients were not zero (0).

Table 7: Coefficients ${ }^{a}$

\begin{tabular}{|c|c|c|c|c|c|c|}
\hline & \multirow{2}{*}{ Model } & \multicolumn{2}{|c|}{ Unstandardized Coefficients } & $\begin{array}{c}\text { Standardized } \\
\text { Coefficients }\end{array}$ & \multirow[t]{2}{*}{$t$} & \multirow{2}{*}{ Sig. } \\
\hline & & $B$ & Std. Error & Beta & & \\
\hline \multirow{3}{*}{1} & (Cons: Present basic pay) & $-38670.051\left(B_{0}\right)$ & 6422.814 & & -6.021 & 0.000 \\
\hline & Gender & $554.461\left(B_{1}\right)$ & 1024.262 & 0.014 & 0.541 & 0.589 \\
\hline & Age & $709.891\left(\mathrm{~B}_{2}\right)$ & 86.209 & 0.461 & 8.235 & 0.000 \\
\hline
\end{tabular}




\begin{tabular}{|c|c|c|c|c|c|}
\hline Marital Status & $-5124.611\left(\mathrm{~B}_{3}\right)$ & 3613.823 & -0.036 & -1.418 & 0.157 \\
\hline Religion & $1360.314\left(\mathrm{~B}_{4}\right)$ & 1118.681 & 0.031 & 1.216 & 0.225 \\
\hline Present designation & $6201.641\left(\mathrm{~B}_{5}\right)$ & 461.322 & 0.571 & 13.443 & 0.000 \\
\hline First joining post & $1902.867\left(\mathrm{~B}_{6}\right)$ & 374.125 & 0.256 & 5.086 & 0.000 \\
\hline Type of Bank & $1834.212\left(\mathrm{~B}_{7}\right)$ & 1621.950 & 0.051 & 1.131 & 0.259 \\
\hline Name of the Bank & $282.551\left(\mathrm{~B}_{8}\right)$ & 289.017 & 0.045 & 0.978 & 0.329 \\
\hline Location (District) & $-1672.287\left(\mathrm{~B}_{9}\right)$ & 1695.606 & -0.078 & -0.986 & 0.325 \\
\hline Location (Upazilla) & $74.986\left(\mathrm{~B}_{10}\right)$ & 250.856 & 0.024 & 0.299 & 0.765 \\
\hline Educational qualifi. & $1623.504\left(\mathrm{~B}_{11}\right)$ & 656.077 & 0.085 & 2.475 & 0.014 \\
\hline Group/ Subject & $-323.377\left(\mathrm{~B}_{12}\right)$ & 525.967 & -0.016 & -0.615 & 0.539 \\
\hline Banking Diploma & $878.569\left(\mathrm{~B}_{13}\right)$ & 569.666 & 0.047 & 1.542 & 0.124 \\
\hline
\end{tabular}

The fitted regression model can be defined as,Model:

Present Basic Salary $=$

$-38670.051 *($ Constant $)+554.461 *$ Gender $+709.891 *$ Age $-5124.611 *$ MaritalStatus $+1360.314 *$

Religion $+6201.641 *$ Presentdesignation $+1902.867 *$ Firstjoiningdesignation $+1834.212 *$ TypeofBank + $282.551 *$ NameoftheBank $-1672.287 *$ Location(Disrtict) $+74.986 *$ Location(Upazilla) $+1623.504 *$

Educationalqualification $-323.377 *$ GrouporSubject $+878.569 *$ BankingDiploma

Remarks: From the fitted (Table 7) the age, present designation, first joins designation, educational qualification were statistically significance at $5 \%$ level of significance. Among the significant independent predictors age (709.891), present designation (6201.641) and first joining designation/post (1902.867) were positive contribution to the dependent variable present basic salary.
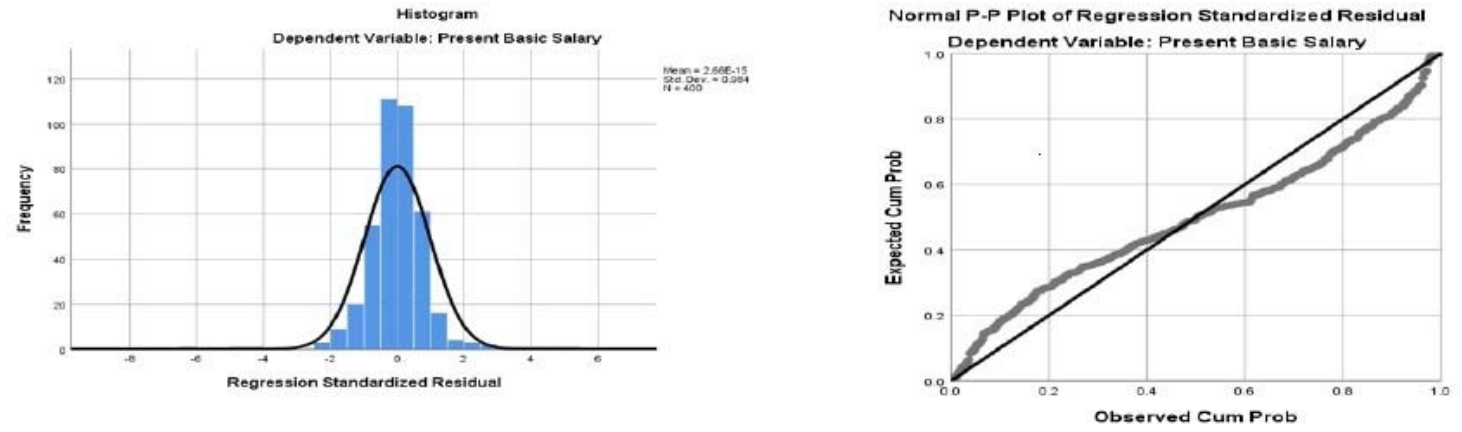

Figure 3 \& 4: Histogram and P-P Plot of Regression Standardized Residual (Present basic salary)

Remarks: From the fitted histogram (Figure $3 \& 4$ ) showed the bell shape curve and $\mathrm{Q}-\mathrm{Q}$ (quantilequantile) plot the fitted residual line passing through the origin. Therefore, the dependent variable Present Basic Salary is normally distributed and the linear regression model best fit for this dataset.

Table 8: Regression-Model Summary

\begin{tabular}{|c|c|c|c|c|c|c|c|c|c|}
\hline \multirow[b]{2}{*}{ Model } & \multirow[b]{2}{*}{$\mathrm{R}$} & \multirow[b]{2}{*}{$\mathrm{R}^{2}$} & \multirow[b]{2}{*}{$\begin{array}{c}\text { Adjusted } \\
\mathrm{R}^{2}\end{array}$} & \multirow{2}{*}{$\begin{array}{l}\text { Std. Err. of } \\
\text { the } \\
\text { Estimates }\end{array}$} & \multicolumn{5}{|c|}{ Change Statistics } \\
\hline & & & & & $\mathrm{R}^{2}$ & $\begin{array}{c}\mathrm{F} \\
\text { change }\end{array}$ & $\mathrm{df1}$ & $\mathrm{df2}$ & Sig. $F$ \\
\hline 1 & $0.826^{a}$ & 0.682 & 0.672 & 15365.25 & 0.682 & 63.742 & 13 & 386 & 0.000 \\
\hline $\begin{array}{l}\text { a. Pred } \\
\text { Locatic }\end{array}$ & $\begin{array}{l}\text { s: (Cons } \\
\text { istrict), } 7\end{array}$ & $\begin{array}{l}\text {, Banki } \\
\text { of Ban }\end{array}$ & $\begin{array}{l}\text { oloma, G } \\
\text { sent Des } \\
\end{array}$ & $\begin{array}{l}\text { Subject, Ger } \\
\text { ion, First joini } \\
\text { (Upazilla) }\end{array}$ & $\begin{array}{l}\text { Religic } \\
\text { designa }\end{array}$ & $\begin{array}{l}\text { ducationa } \\
\text { post, Nam }\end{array}$ & ualific & $\begin{array}{l}\mathrm{n}, \mathrm{Ma} \\
\mathrm{Cat}\end{array}$ & $\begin{array}{l}\text { Status, } \\
\text { ccation }\end{array}$ \\
\hline
\end{tabular}

Remarks: The fitted linear regression model (Table 8) for Present Gross Salary and other independent variables listed above. The model is good fit for this dataset and the coefficient of multiple determinations $R^{2}$ is 0.682 .
Since the $R^{2} \sim 0.682$ then the dependent variable present gross salary $68.2 \%$ explained by the independent variables/predictors. 
Table 9: ANOVA $^{\mathrm{a}}$

\begin{tabular}{|c|c|c|c|c|c|c|}
\hline & Ddel & Sum of Squares & df & Mean Square & $\mathrm{F}$ & Sig. \\
\hline 1 & Regression & 195635872639.298 & 13 & 15048913279.950 & 63.742 & $0.000^{\mathrm{b}}$ \\
\hline & Residual & 91131097360.703 & 386 & 236090925.805 & & \\
\hline & Total & 286766970000.000 & 399 & & & \\
\hline & $\overline{\text { Dependent }}$ & riable: Present gross & salary & & & \\
\hline & $\begin{array}{l}\text { Predictors: } \\
\text { ucational Q } \\
\text { signation, } \\
\text { pazilla) }\end{array}$ & $\begin{array}{l}\text { nstant), Banking } \\
\text { cation, Marital Stat } \\
\text { joining designatio }\end{array}$ & iplom & $\begin{array}{l}\text { Group/ Subjec } \\
\text { ion (District), Ty } \\
\text { Jame of the }\end{array}$ & ender & $\begin{array}{l}\text { ligion, } \\
\text { resent } \\
\text { cation }\end{array}$ \\
\hline
\end{tabular}

Hypothesis: The null hypothesis, $\mathrm{HO}: \mathrm{B}_{0}=\mathrm{B}_{1}=\mathrm{B}_{2}=\ldots \ldots \ldots=\mathrm{B}_{13}=0$

The alternative hypothesis, $\mathrm{H1}: \mathrm{B}_{0}=\mathrm{B}_{1}=\mathrm{B}_{2}=\ldots \ldots \ldots=\mathrm{B}_{13} \neq 0$

Remarks: From the (Table 9) ANOVA (Analysis of Variance) table the fitted regression model F-test statistic value is 63.742 and the significance value $(p$ value) 0.000 . Then all the regression coefficients were statistically highly significant at $5 \%$ level of significance. That is, alternative hypothesis is accepted. Therefore, all the regression coefficients were not zero (0).

Table 10: Coefficients ${ }^{a}$

\begin{tabular}{|c|c|c|c|c|c|c|}
\hline \multirow{2}{*}{\multicolumn{2}{|c|}{ Model }} & \multicolumn{2}{|c|}{ Unstandardized Coefficients } & \multirow{2}{*}{$\begin{array}{c}\text { Standardized } \\
\text { Coefficients } \\
\text { Beta } \\
\end{array}$} & \multirow{2}{*}{$\mathrm{t}$} & \multirow{2}{*}{ Sig. } \\
\hline & & B & Std. Error & & & \\
\hline \multirow{14}{*}{1} & $\begin{array}{c}\text { (Constant: Present } \\
\text { gross) }\end{array}$ & -76402.124 & 14122.791 & & -5.410 & 0.000 \\
\hline & Gender & 1172.807 & 2252.196 & 0.016 & 0.521 & 0.603 \\
\hline & Age & 1244.412 & 189.561 & 0.432 & 6.565 & 0.000 \\
\hline & Marital Status & -9286.091 & 7946.247 & -0.035 & -1.169 & 0.243 \\
\hline & Religion & 1028.259 & 2459.809 & 0.012 & 0.418 & 0.676 \\
\hline & Present designation & 10226.033 & 1014.378 & 0.503 & 10.081 & 0.000 \\
\hline & First joining design. & 3129.422 & 822.645 & 0.225 & 3.804 & 0.000 \\
\hline & Type of Bank & 15523.420 & 3566.423 & 0.232 & 4.353 & 0.000 \\
\hline & Name of the Bank & 420.502 & 635.505 & 0.036 & 0.662 & 0.509 \\
\hline & Location (District) & -4565.112 & 3728.379 & -0.113 & -1.224 & 0.222 \\
\hline & Location (Upazilla) & 326.900 & 551.594 & 0.057 & 0.593 & 0.554 \\
\hline & $\begin{array}{l}\text { Educational } \\
\text { qualification }\end{array}$ & 2791.159 & 1442.615 & 0.078 & 1.935 & 0.054 \\
\hline & Group/Subject & -282.423 & 1156.521 & -0.007 & -0.244 & 0.807 \\
\hline & Banking Diploma & 1700.733 & 1252.610 & 0.048 & 1.358 & 0.175 \\
\hline & & a. D & iable: $\mathrm{F}$ & salary & & \\
\hline
\end{tabular}

The fitted regression model can be defined as,

Present Gross Salary

$$
\begin{aligned}
& =-76402.124 *(\text { Constant })+1172.807 * \text { Gender }+1244.412 * \text { Age }-9286.091 * \text { MaritalStatus } \\
& +1028.259 * \text { Religion }+10226.033 * \text { PresentDesignation }+3129.422 * \text { Firstjoiningdesignation } \\
& +15523.420 * \text { Typeof Bank }+420.502 * \text { NameoftheBank }-4565.112 * \text { Location }(\text { Disrtict }) \\
& +326.900 * \text { Location }(\text { Upazilla })+2791.159 * \text { EducationalQualification }-282.423 \\
& * \text { GrouporSubject }+1700.733 * \text { BankingDiploma }
\end{aligned}
$$

Remarks: From the (Table 10) fitted the age, present designation, first joins designation, educational qualification were statistically significance at $5 \%$ level of significance. Among the significant independent predictors age (1244.412), present designation (10226.033), first joining designation/post (3129.422) and type of bank (15523.420) were positive contribution to the dependent variable Present Gross Salary. Therefore, there is a strong association among age, present designation, joining post and type of bank that affect employee satisfaction. 

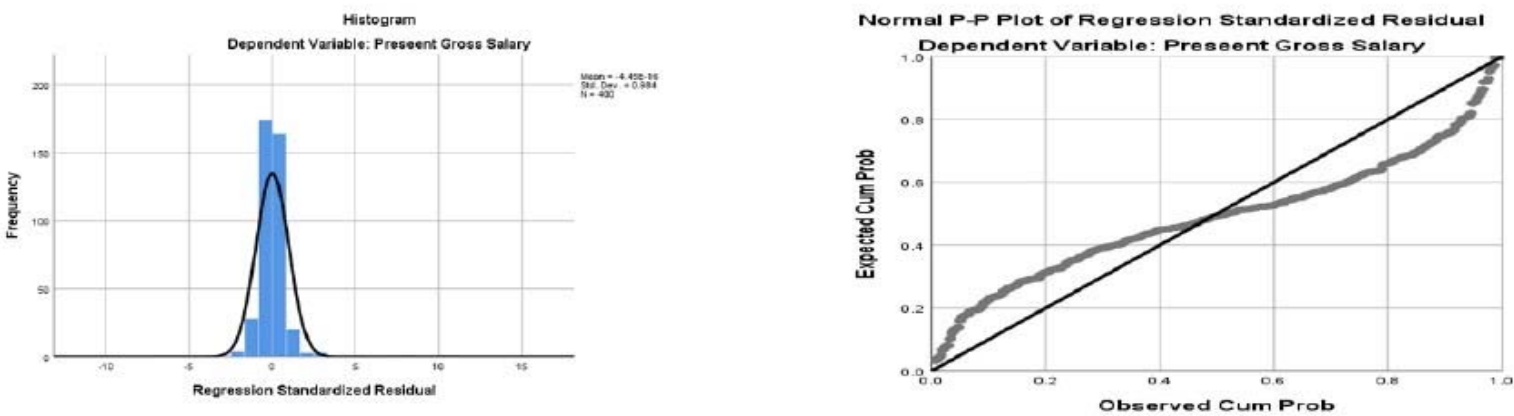

Figure 5 \& 6: Histogram and P-P Plot of Regression Standardized Residual (Present gross salary)

Remarks: From the fitted histogram (Figure $5 \& 6$ ) showed the bell shape curve and $\mathrm{Q}-\mathrm{Q}$ (quantilequantile) plot the fitted residual line passing through the origin. Therefore, the dependent variable Present Gross Salary is normally distributed and the linear regression model best fit for this dataset.

Table 11: Regression-Model Summary ${ }^{b}$

\begin{tabular}{|c|c|c|c|c|c|c|c|c|c|}
\hline \multirow[b]{2}{*}{ Model } & \multirow[b]{2}{*}{$\mathrm{R}$} & \multirow[b]{2}{*}{$\mathrm{R}^{2}$} & \multirow[b]{2}{*}{$\begin{array}{l}\text { Adjusted } \\
\mathrm{R}^{2}\end{array}$} & \multirow[b]{2}{*}{$\begin{array}{l}\text { Std. Err. of } \\
\text { the Estimates }\end{array}$} & \multicolumn{5}{|c|}{ Change Statistics } \\
\hline & & & & & $\mathrm{R}^{2}$ & $\begin{array}{c}\mathrm{F} \\
\text { change }\end{array}$ & df1 & df2 & Sig. F \\
\hline 1 & $0.689^{a}$ & 0.475 & 0.457 & 5713.00316 & 0.475 & 26.857 & 13 & 386 & 0.000 \\
\hline $\begin{array}{l}\text { a. Predi } \\
\text { Locatio }\end{array}$ & $\begin{array}{l}\text { :(Cons } \\
\text { istrict), }\end{array}$ & $\begin{array}{l}\text { Bank } \\
\text { of Bar }\end{array}$ & $\begin{array}{l}\text { Diploma, Gr } \\
\text { Present Desic }\end{array}$ & $\begin{array}{l}\text { p/ Subject, Gend } \\
\text { ation, First joining } \\
\text { (Upazilla). }\end{array}$ & $\begin{array}{l}\text { Religion } \\
\text { esignatic }\end{array}$ & $\begin{array}{l}\text { ducationa } \\
\text { ost, Nam }\end{array}$ & $\begin{array}{l}\text { Ialifice } \\
\text { the E }\end{array}$ & $\begin{array}{l}\text {, Mari } \\
\text {, Age, }\end{array}$ & $\begin{array}{l}\text { Status, } \\
\text { cation }\end{array}$ \\
\hline
\end{tabular}

Remarks: The fitted linear regression model (Table 11) for Initial Basic Salary and other independent variables listed above. The model is fit for this dataset and the coefficient of multiple determinations $R^{2}$ is 0.475 . Since the $R^{2} \sim 0.475$ then the dependent variable initial basic salary $47.5 \%$ explained by the independent estimators.

\section{Table 12: ANOVA ${ }^{a}$}

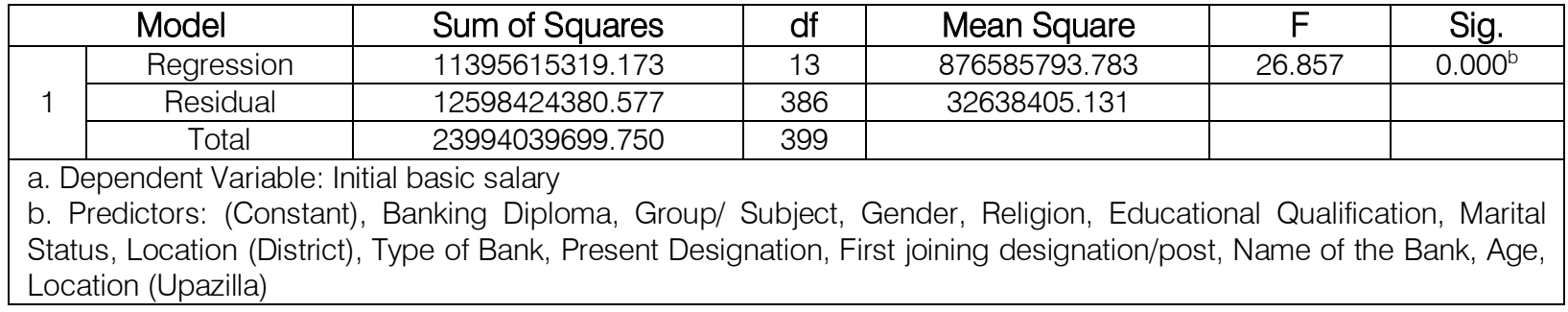

Hypothesis: The null hypothesis, $\mathrm{HO}: \mathrm{B}_{0}=\mathrm{B}_{1}=\mathrm{B}_{2}=\ldots \ldots \ldots=\mathrm{B}_{13}=0$

The alternative hypothesis, $\mathrm{H} 1: \mathrm{B}_{0}=\mathrm{B}_{1}=\mathrm{B}_{2}=\ldots \ldots \ldots=\mathrm{B}_{13} \neq 0$

Remarks: From the ANOVA Table 12 the fitted level of significance. That is the null hypothesis is regression model F-test statistic value is 26.857 and the rejected and alternative hypothesis is accepted. significance value ( $p$ value) 0.000 . Then all the Therefore, all the regression coefficients were not zero regression coefficients were statistically significant at $5 \%$ (0).

Table 13: Coefficients ${ }^{\mathrm{a}}$

\begin{tabular}{|c|c|c|c|c|c|c|}
\hline & \multirow{2}{*}{ Model } & \multicolumn{2}{|c|}{ Unstandardized Coefficients } & \multirow{2}{*}{$\begin{array}{c}\begin{array}{c}\text { Standardized } \\
\text { Coefficients }\end{array} \\
\text { Beta }\end{array}$} & \multirow[t]{2}{*}{$\mathrm{t}$} & \multirow{2}{*}{ Sig. } \\
\hline & & $B$ & Std. Error & & & \\
\hline \multirow{5}{*}{1} & (Constant: Initial Basic Pay) & 6234.435 & 5244.365 & & 1.189 & 0.235 \\
\hline & Gender & -350.697 & 838.100 & -0.017 & -0.418 & 0.676 \\
\hline & Age & -121.912 & 71.022 & -0.146 & -1.717 & 0.087 \\
\hline & Marital Status & 1247.972 & 2977.753 & 0.016 & 0.419 & 0.675 \\
\hline & Religion & -21.884 & 918.839 & -0.001 & -0.024 & 0.981 \\
\hline
\end{tabular}




\begin{tabular}{|c|c|c|c|c|c|}
\hline Present Designation & -671.561 & 378.451 & -0.114 & -1.775 & 0.077 \\
\hline First joining designation/post & 2220.853 & 308.118 & 0.551 & 7.208 & 0.000 \\
\hline Type of Bank & -1285.682 & 1402.737 & -0.066 & -0.917 & 0.360 \\
\hline Name of the Bank & 1064.391 & 236.772 & 0.326 & 4.495 & 0.000 \\
\hline Location (District) & 1761.002 & 1401.011 & 0.162 & 1.257 & 0.210 \\
\hline Location (Upazilla) & -280.441 & 206.022 & -0.178 & -1.361 & 0.174 \\
\hline Educational Qualification & -879.276 & 538.907 & -0.085 & -1.632 & 0.104 \\
\hline Group/ Subject & -306.230 & 431.933 & -0.027 & -0.709 & 0.479 \\
\hline Banking Diploma & -199.290 & 462.560 & -0.020 & -0.431 & 0.667 \\
\hline \multicolumn{7}{|l|}{ a. Dependent Variable: Initial basic salary } \\
\hline
\end{tabular}

The fitted regression model can be defined as,

Initial basic salary

$$
\begin{aligned}
& =6234.435 *(\text { Constant })-350.679 * \text { Gender }-121.912 * \text { Age }+1247.972 * \text { Marital Status } \\
& -21.884 * \text { Religion }-671.561 * \text { Present designation }+2220.583 * \text { First joining designation } \\
& -1285.682 * \text { Type of Bank }+1064.391 * \text { Name of the Bank }+1761.002 * \text { Location }(\text { Disrtict) } \\
& -280.441 * \text { Location }(\text { Upazilla })-879.276 * \text { Educational qualification }-306.230 \\
& * \text { Group or Subject }-199.290 * \text { Banking Diploma }
\end{aligned}
$$

Comment: From the fitted coefficients (Table 13) the, first name of the bank were statistically significance at $5 \%$ level of significance. Among the significant independent predictors first joining designation/post
(2220.853) and name of bank (1064.391) were positive contribution to the dependent variable Initial Basic Salary.

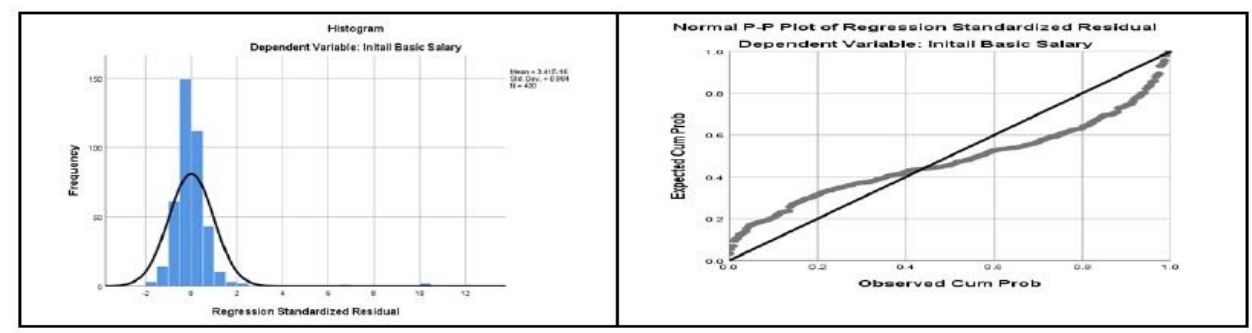

Figure 7 \& 8: Histogram and P-P Plot of Regression Standardized Residual (Initial basic salary)

Remarks: From the Figure $7 \& 8$ depict that the fitted histogram showed the bell shape curve and $Q-Q$ (quantile-quantile) plot the fitted residual line passing

\begin{tabular}{|c|c|c|c|c|c|c|c|c|c|}
\hline \multirow[b]{2}{*}{ Model } & \multirow[b]{2}{*}{$\mathrm{R}$} & \multirow[b]{2}{*}{$\mathrm{R}^{2}$} & \multirow[b]{2}{*}{ Adjusted $\mathrm{R}^{2}$} & \multirow[b]{2}{*}{$\begin{array}{l}\text { Std. Err. of the } \\
\text { Estimates }\end{array}$} & \multicolumn{5}{|c|}{ Change Statistics } \\
\hline & & & & & $\mathrm{R}^{2}$ & $\begin{array}{c}F \\
\text { change }\end{array}$ & df1 & $\mathrm{df} 2$ & Sig. $F$ \\
\hline 1 & $0.592^{\mathrm{a}}$ & 0.351 & 0.329 & 13459.34450 & 0.351 & 16.058 & 13 & 386 & 0.000 \\
\hline
\end{tabular}

through the origin. Therefore, the dependent variable initial basic salary is normally distributed and the linear regression model best fit for this dataset.

Table 14: Regression-Model Summary

\begin{tabular}{|c|c|c|c|c|c|c|}
\hline \multicolumn{2}{|r|}{ Model } & Sum of Squares & $\mathrm{df}$ & Mean Square & $\mathrm{F}$ & Sig. \\
\hline \multirow{3}{*}{1} & Regression & 37815785084.065 & 13 & 2908906544.928 & 16.058 & $0.000^{b}$ \\
\hline & Residual & 69925426394.726 & 386 & 181153954.390 & & \\
\hline & Total & 107741211478.791 & 399 & & & \\
\hline & $\begin{array}{l}\text { ndent Variab } \\
\text { ctors: (Cons } \\
\text { Location (Dis } \\
\text { n (Upazilla) }\end{array}$ & $\begin{array}{l}\text { ross salary } \\
\text { ing Diploma, Gro } \\
\text { of Bank, Present }\end{array}$ & & ing designatic & ne of & nk, Age, \\
\hline
\end{tabular}

a. Predictors: (Constant), Banking Diploma, Group/ Subject, Gender, Religion, Educational Qualification, Marital Status, Location (District), Type of Bank, Present Designation, First joining designation/post, Name of the Bank, Age, Location (Upazilla)

Table 15: ANOVAa

Hypothesis: The null hypothesis, $\mathrm{HO}: \mathrm{B}_{0}=\mathrm{B}_{1}=\mathrm{B}_{2}=\ldots \ldots \ldots=\mathrm{B}_{13}=0$

The alternative hypothesis, $\mathrm{H} 1$ : $\mathrm{B}_{0}=\mathrm{B}_{1}=\mathrm{B}_{2}=\ldots \ldots \ldots=\mathrm{B}_{13} \neq 0$ 
Remarks: From the Table 15 (ANOVA) the fitted regression model F-test statistic value is 16.058 and the significance value ( $p$ value) 0.000 . Then all the regression coefficients were statistically significant at $5 \%$ level of significance. That is the null hypothesis is rejected. Therefore, all the regression coefficients were not zero (0).

Table 16: Coefficients ${ }^{a}$

\begin{tabular}{|c|c|c|c|c|c|c|}
\hline \multirow{2}{*}{\multicolumn{2}{|c|}{ Model }} & \multicolumn{2}{|c|}{$\begin{array}{c}\text { Unstandardized } \\
\text { Coefficients }\end{array}$} & \multirow{2}{*}{$\begin{array}{c}\begin{array}{c}\text { Standardized } \\
\text { Coefficients }\end{array} \\
\text { Beta } \\
\end{array}$} & \multirow[t]{2}{*}{$\mathrm{t}$} & \multirow[t]{2}{*}{ Sig. } \\
\hline & & $B$ & Std. Error & & & \\
\hline \multirow{14}{*}{1} & (Constant: Initial Gross Pay) & 181.712 & 12371.000 & & 0.015 & 0.988 \\
\hline & Gender & 25.992 & 1972.834 & 0.001 & 0.013 & 0.989 \\
\hline & Age & -90.130 & 166.047 & -0.051 & -0.543 & 0.588 \\
\hline & Marital Status & 5525.183 & 6960.595 & 0.033 & 0.794 & 0.428 \\
\hline & Religion & -251.863 & 2154.695 & -0.005 & -0.117 & 0.907 \\
\hline & Present designation & -1829.536 & 888.554 & -0.147 & -2.059 & 0.040 \\
\hline & First joining designation & 4425.217 & 720.604 & 0.519 & 6.141 & 0.000 \\
\hline & Type of Bank & -1458.181 & 3124.043 & -0.036 & -0.467 & 0.641 \\
\hline & Name of the Bank & 2449.083 & 556.677 & 0.337 & 4.399 & 0.000 \\
\hline & Location (District) & 2369.895 & 3265.911 & 0.096 & 0.726 & 0.468 \\
\hline & Location (Upazilla) & -360.575 & 483.174 & -0.102 & -0.746 & 0.456 \\
\hline & Educational qualification & -1895.923 & 1263.673 & -0.086 & -1.500 & 0.134 \\
\hline & Group/Subject & 164.586 & 1013.066 & 0.007 & 0.162 & 0.871 \\
\hline & Banking Diploma & 321.652 & 1097.236 & 0.015 & 0.293 & 0.770 \\
\hline \multicolumn{7}{|c|}{ a. Dependent Variable: Initial Gross Salary } \\
\hline \multicolumn{7}{|c|}{$\begin{array}{l}\text { From the coefficients Table } 16 \text { the study observed that present designation, first joining and name of the bank were statistically } \\
\text { significance at } 5 \% \text { level of significance. Among the significant independent predictors first joining designation/post (4425.217)and } \\
\text { name of bank (2449.083) were positively affected the dependent variable Initial Basic Salary. }\end{array}$} \\
\hline
\end{tabular}

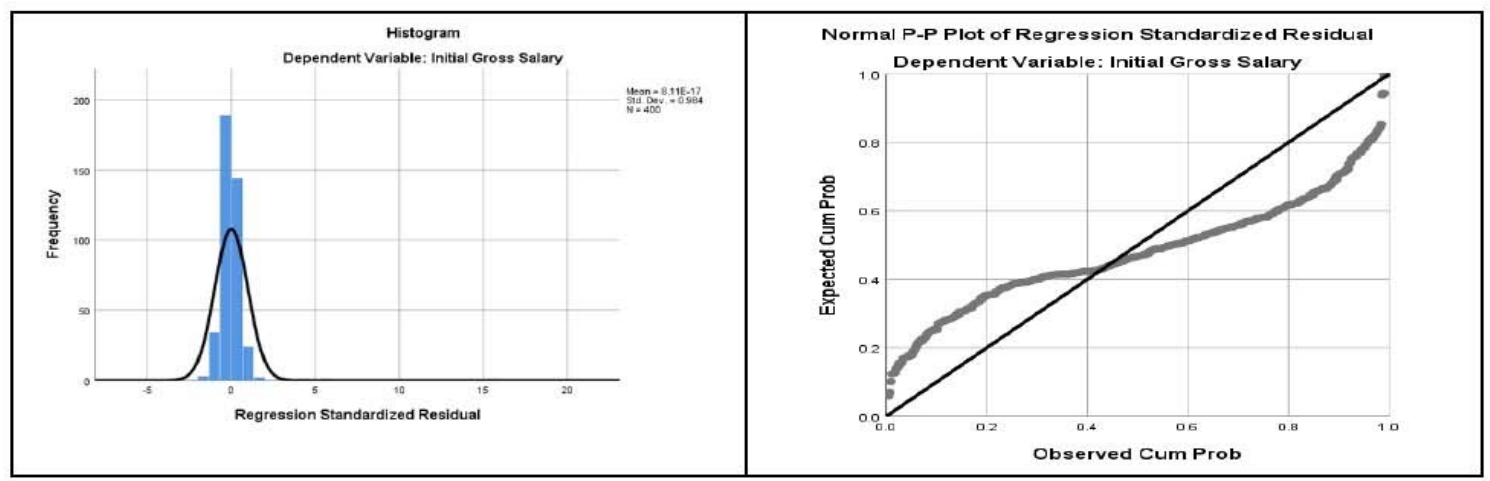

Figure 9 \& 10: Histogram and P-P Plot of Regression Standardized Residual (Initial gross pay)

Remarks: From the fitted histogram (Figure $9 \& 10$ ) showed the bell shape curve and $Q-Q$ (quantilequantile) plot the fitted residual line passing through the origin. Therefore, the dependent variable initial gross salary is normally distributed and the linear regression model best fit for this dataset.

Table 17: Results of alternative hypotheses $\left(H_{a}\right)$ from ANOVA Table 3,6,9,12, \&16

\begin{tabular}{|l|l|l|l|}
\hline$H_{a}$ & Results & Remarks & Relationship/ effect on employee motivation \\
\hline$H_{a 1}$ & $p<0.05$ & Sustained & Employee motivation is related to work experience. \\
\hline$H_{a 2}$ & $p<0.05$ & Sustained & There is a relationship between motivation present basic pay. \\
\hline $\mathrm{H}_{\mathrm{a} 2}$ & $\mathrm{p}<0.05$ & Sustained & Present gross salary affects employee motivation. \\
\hline $\mathrm{H}_{\mathrm{a} 1}$ & $\mathrm{p}<0.05$ & Sustained & Initial gross salary motivates employee positively. \\
\hline $\mathrm{H}_{\mathrm{a} 1}$ & $\mathrm{p}<0.05$ & Sustained & Initial basic salary motivates employees positively. \\
\hline
\end{tabular}


Overall it can be explained that employees are in the service of the banking sector influenced by the age, experience, present designation, joining post, type of bank, present and initial gross. There were strong relationships among these control or sources variables in the study. Gender did not affect the satisfaction of the bank employees. Among these factors the most effective factors were age and present designation (standardized Beta coefficient value 0.663 and 0.571). On the other hand, banking diploma mostly affects the promotion in case of public bank.

Table 18: Overall findings from the Table 2\&4, 5\&7, 8\&10, 11\&13, 14\&16, 17

\begin{tabular}{|c|c|c|c|c|}
\hline Dependent variable & $\mathrm{R}^{2}$ & Standardized coefficient & Effective factors & Motivation \\
\hline Work experience & $94 \%$ & $\begin{array}{c}\text { Age }(\beta=0.663) \text {, present designation } \\
(\beta=0.152)\end{array}$ & Age & \multirow{5}{*}{ 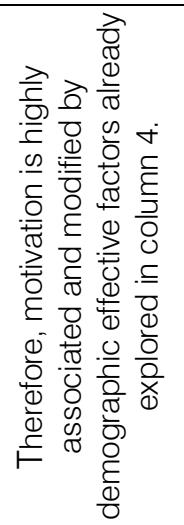 } \\
\hline Present basic pay & $77 \%$ & $\begin{array}{c}\text { Present designation }(\beta=0.571) \text {, age } \\
(\beta=0.461) \text {, and first joining } \\
\text { designation }(\beta=0.256)\end{array}$ & Present designation & \\
\hline Present gross pay & $68.2 \%$ & $\begin{array}{c}\text { Present designation }(\beta=0.503) \text {, age } \\
(\beta=0.432), \text { type of bank } \\
(\beta=0.232) \text { and first joining } \\
\text { designation }(\beta=0.225)\end{array}$ & Present designation & \\
\hline Initial basic pay & $47.5 \%$ & $\begin{array}{c}\text { First joining designation }(\beta=0.551) \text {, } \\
\text { and Name of the bank }(\beta=0.326)\end{array}$ & $\begin{array}{l}\text { First joining } \\
\text { designation }\end{array}$ & \\
\hline Initial gross pay & $35.1 \%$ & $\begin{array}{c}\text { First joining designation }(\beta=0.519) \text {, } \\
\text { and Name of the bank }(\beta=0.337)\end{array}$ & $\begin{array}{l}\text { First joining } \\
\text { designation }\end{array}$ & \\
\hline
\end{tabular}

It is evident from the Table 18 work experience is $94 \%$ explained by variance whereas age is strongly associated with job experience. Present basic salary $77 \%$ is explained by total predictors and it is highly affected by present designation of the existing employees. Present gross salary $68.2 \%$ is varied by the estimators and it is mostly influenced by present position of an employee. Initial basic salary is $47.5 \%$ $\sim 48 \%$ explained by variance and is prejudiced by first joining designation. Initial gross salary $35.1 \%$ is explained by total variance and it is strongly affected by employees' first joining designation. Therefore, employees' motivation of the banking sector of Bangladesh is highly associated with work experience, present and initial salaries which are mostly modified by age, present and initial designation. Finally, equation may be Employee Motivation $=f($ Age + Present designation + Initial designation).

\section{Concluding Remarks}

Motivation is a complex to determine its level. The study found from the empirical analysis based on demographic outlined that the most effective factors age, present designation, first joining designation were explored and strongly associated with employee motivation in the banking sector of Bangladesh. Sample area, job experience and pay were considered only which may confine the scope of the study. Lack of huge context of the research is another limitation in the paper. Further study should emphasize in a larger scale of employee perception concentrating on job security and money or pay intrinsically as well as extrinsically.

\section{ACKNOWLEDGEMENT}

The author of this article is grateful to the honorable Professor Dr. Md. Omar Faruk Sarker (PhD Supervisor of the author), Marketing Department, \& Dr. Md. Kamruzzaman (PhD Co-Supervisor of the author), Associate Professor (Statistics), IBS, University of Rajshahi, Bangladesh.

\section{References Références Referencias}

1. Alderfer, C., P. (1969). An empirical test of a new theory of human needs. Organizational Behavior and Human Performance. 4 (2): 142-75. doi: 10.1016/003 0-5073(69)90004-X.

2. BRPD Circular Letter No. 07, Dated 17 June 2015.

3. Dawson, K. M. and Dawson, S. N. (1991). The cure for employee malaise-motivation. Clinical Laboratory Management Review, Vol. 5 No. 4: 296-98, 300, 302.

4. Endress P. (2007). How to hire and retain top talent, management corner: 10-11.

5. Gordon, G. G (1965). The relationship of satisfiers and dissatisfiers to productivity, turnover and morale, American Psychologist, Vol. 20: 499-502.

6. Griffin, R. W. (1990), Management, 3rd ed., Houghton Mifflin Company, Dallas, TX: 437.

7. Herzberg, F. (1987). Workers' needs: the same around the world. Industry Week: 29-30 32.

8. Islam, M., T., Haque, M., M., Yesmin, R., Ismal, M., R., and Islam, M.,M. (2017). Human Resource of Banks. Banking Review Series, BIBM, Dhaka: 292.

9. Kovach, K., A. (1987). What motivates employees? Workers and supervisors give different answers. Business Horizons, Vol. 30(5): 58-65. 
10. Maslow, A. H. (1954). Motivation and Personality: NY: Harper.

11. Mc Clellend, D. (1961). The Achieving Society. NY: The Free Press.

12. Nyameh Jerome, N. (2013). Application of the Maslow's hierarchy of need theory; impacts and implications on organizational culture, human resource and employee's performance. International Journal of Business and Management Invention, Vol. 2 (3): 39-45.

13. Wiley, C. (1997). What motivates employees according to over 40 years motivation surveys. International Journal of Manpower, Vol. 18, Issue 3:263-280; http://dx.do.org/10-1108/0143772971 0169373.

14. www. ventureline.com 